New windows on massive stars: asteroseismology, interferometry, and spectropolarimetry

Proceedings IAU Symposium No. 30\%, 2014

G. Meynet, C. Georgy, J. H. Groh 83 Ph. Stee, eds.

(C) International Astronomical Union 2015 doi:10.1017/S1743921314006450

\title{
Discrete absorption components in the massive LBV Binary MWC 314
}

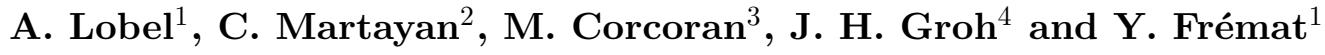 \\ ${ }^{1}$ Royal Observatory of Belgium, Brussels \\ email: Alex.Lobel@oma.be, alobel@sdf.org \\ ${ }^{2}$ European Southern Observatory, Chile \\ ${ }^{3}$ Goddard Space Flight Center, Greenbelt MD, USA \\ ${ }^{4}$ Geneva Observatory, Versoix, Switzerland
}

\begin{abstract}
We investigate the physical properties of large-scale wind structures around massive hot stars with radiatively-driven winds. We observe Discrete Absorption Components (DACs) in optical He I P Cygni lines of the LBV binary MWC $314\left(P_{\text {orb }}=60.8 \mathrm{~d}\right)$. The DACs are observed during orbital phases when the primary is in front of the secondary star. They appear at wind velocities between $-100 \mathrm{~km} \mathrm{~s}^{-1}$ and $-600 \mathrm{~km} \mathrm{~s}^{-1}$ in the $\mathrm{P}$ Cyg profiles of HeI $\lambda 5875, \lambda 6678$, and $\lambda 4471$, signaling high-temperature expanding wind regions of enhanced density and variable outflow velocity. The DACs can result from wave propagation linked to the orbital motion near the low-velocity wind base. The He I lines indicate DAC formation close to the primary's surface in high-temperature wind regions in front of its orbit, or in dynamical wind regions confined between the binary stars. We observed the DACs with Mercator-HERMES on 5 Sep 2009, 5 May 2012, and 6 May 2014 when the primary is in front of the secondary star. XMM-Newton observations of 6 May 2014 significantly detected MWC 314 in X-rays at an average rate of $\sim 0.015 \mathrm{cts} \mathrm{s}^{-1}$.
\end{abstract}

Keywords. stars: winds, outflows, emission-line, Be; binaries: eclipsing; X-rays

\section{Introduction}

Current understanding of the role of the Luminous Blue Variable (LBV) stage in the evolution of the most massive stars is very limited. Important questions are how the eruptions of several $M_{\odot}$ are triggered, and what role binarity plays in the properties of the LBV stage and in shaping the nebulae observed around LBVs. For example, is most of the mass lost by an LBV star due to a steady radiatively-driven stellar wind, or is the H-rich envelope removed by punctuated eruption-driven mass loss? There are two confirmed LBV binaries: $\eta$ Car in the Galaxy, and HD 5980 in the SMC. The extremely luminous star MWC 314 has recently been recognized $\left(P_{\text {orb }}=60.8 \mathrm{~d}\right.$ and $e=0.23$ ) as an eclipsing massive binary system (Lobel et al. 2013). Indications that MWC 314 is a (dormant) LBV are strong. The star is very luminous $\left(\log L / L_{\odot}=5.9\right.$; see also Miroshnichenko et al. 1998) with an optical spectrum and SED nearly identical to the canonical LBV P Cygni. MWC 314 is surrounded by a bipolar $\mathrm{H} \alpha$ nebula that may be the result of an eruption of MWC 314 more than 100,000 years ago (Marston \& McCollum 2008). MWC 314 is a single-lined, eclipsing binary system with masses of $40+26 M_{\odot}$, and radii of $87+20 R_{\odot}$. The largest diameter of the binary system is $\sim 1.1 \mathrm{AU}$.

\section{Radial velocity curve and DACs in He I P Cyg lines}

We measure the radial velocity curve in Fig. 1 from selected absorption lines of the primary star. Two spectra marked with $\mathrm{A}$ and $\mathrm{C}$ are observed in the quadrature phase 

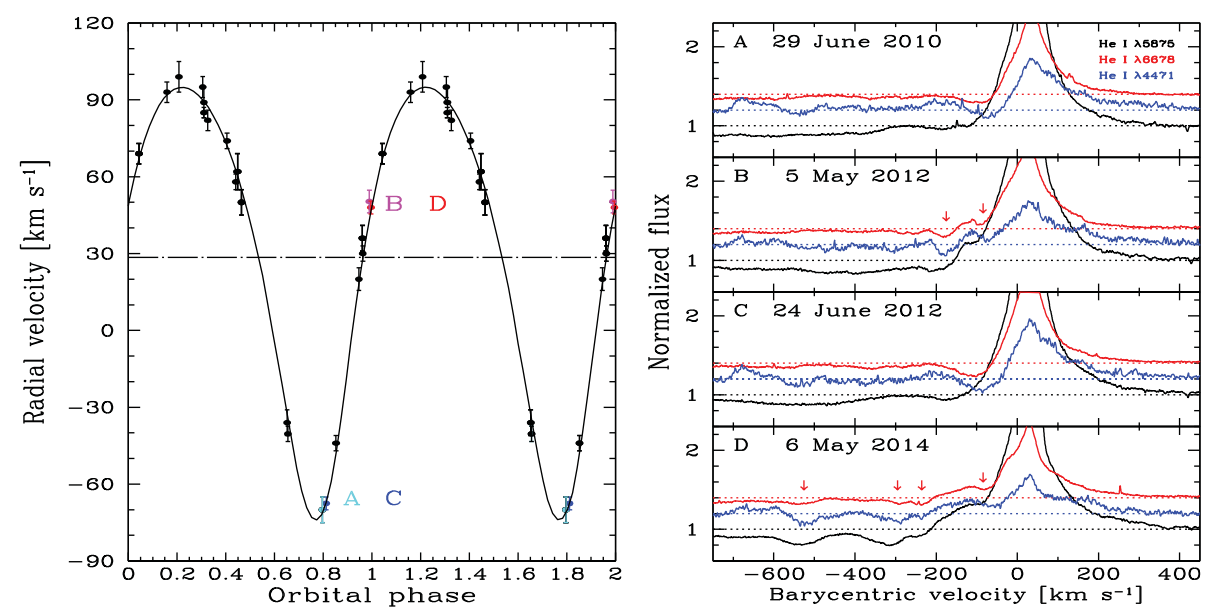

Figure 1. Left-hand panel: orbital radial velocity curve of MWC 314. Two orbital phases $\mathrm{B}$ and $\mathrm{D}$ when the primary is in front of the secondary $(\phi \sim 0)$, and two quadrature phases $\mathrm{A}$ and $\mathrm{C}(\phi \sim 0.8)$ are marked. Right-hand panels: the profiles of three He I lines are shown for orbital phases marked A, B, C, and D. DACs are observed in the violet wings of the lines (marked with arrows) during the orbital phases B and D. XMM-Newton observed X-rays on 6 May 2014 (D).

close to minimum radial velocity of the primary $(0.75<\phi<0.85)$. Two spectra observed when the primary is in front of the secondary are marked with $\mathrm{B}$ and $\mathrm{D}(0.95<\phi<1.05)$. Three He I lines reveal P Cyg profiles with wind expansion velocities to $\sim 1200 \mathrm{~km} \mathrm{~s}^{-1}$. On 5 May 2012 (B) we observe two discrete absorption components in the extended violet wings of the three lines. The DACs are observed at wind velocities of $\sim 100 \mathrm{~km} \mathrm{~s}^{-1}$ and $\sim 180 \mathrm{~km} \mathrm{~s}^{-1}$ in the line profiles (marked with arrows). The DACs are not clearly observed in the quadrature phases of 29 June 2010 (A) and 24 June 2012 (C). We observe four DACs on 6 May 2014 (D) at wind velocities between $\sim 100 \mathrm{~km} \mathrm{~s}^{-1}$ and $\sim 600 \mathrm{~km} \mathrm{~s}^{-1}$.

\section{Conclusions}

Our high-resolution spectroscopic monitoring program of MWC 314 with MercatorHERMES during the last 5 years reveals DACs in the orbital phases when the primary is in front of the secondary star on 5 Sep 2009, 5 May 2012, and 6 May 2014. The high-excitation temperatures of the He I lines signal expanding wind regions of enhanced density and variable outflow velocity. The DACs can form close to the primary's surface (of B0-type) in high-temperature and density-enhanced wind regions in front of its orbit. The recurrence of the DACs in orbital phases when the primary is in front of the secondary can result from wave propagation which is physically linked to the orbital motion near the (low-velocity $<150 \mathrm{~km} \mathrm{~s}^{-1}$ ) wind base. Alternatively, the DACs can form in dynamical (high-temperature) wind regions confined between the binary stars at the shock interface of a colliding wind region. We expect orbital X-ray variability in MWC 314 (similar to the LBVs $\eta$ Car and HD 5980) during planned XMM-Newton observations in the quadrature phase of Oct. 2014.

\section{References}

Lobel, A., Groh, J. H., Martayan, C., et al. 2013, A\& A 559, A16

Marston, A. P. \& McCollum, B. 2008, A $\& A$ 477, 193

Miroshnichenko, A. S., Frémat, Y., Houziaux, L., et al. 1998, A\& $A S$ 131, 469 